\title{
The Chirped-Pulse Inverse Free Electron Laser: A Tabletop, High- Gradient Vacuum Laser Accelerator
}

F.V. Hartemann, A.L. Troha, H.A. Baldis

March 5, 2001

Lawrence

Livermore

National

Laboratory 


\section{DISCLAIMER}

This document was prepared as an account of work sponsored by an agency of the United States Government. Neither the United States Government nor the University of California nor any of their employees, makes any warranty, express or implied, or assumes any legal liability or responsibility for the accuracy, completeness, or usefulness of any information, apparatus, product, or process disclosed, or represents that its use would not infringe privately owned rights. Reference herein to any specific commercial product, process, or service by trade name, trademark, manufacturer, or otherwise, does not necessarily constitute or imply its endorsement, recommendation, or favoring by the United States Government or the University of California. The views and opinions of authors expressed herein do not necessarily state or reflect those of the United States Government or the University of California, and shall not be used for advertising or product endorsement purposes.

This work was performed under the auspices of the U. S. Department of Energy by the University of California, Lawrence Livermore National Laboratory under Contract No. W-7405-Eng-48.

This report has been reproduced directly from the best available copy.

Available to DOE and DOE contractors from the

Office of Scientific and Technical Information

P.O. Box 62, Oak Ridge, TN 37831

Prices available from (423) 576-8401

http://apollo.osti.gov/bridge/

Available to the public from the

National Technical Information Service

U.S. Department of Commerce 5285 Port Royal Rd., Springfield, VA 22161 http://www.ntis.gov/

$$
\text { OR }
$$

Lawrence Livermore National Laboratory Technical Information Department's Digital Library http://www.llnl.gov/tid/Library.html 


\title{
The Chirped-Pulse Inverse Free Electron Laser: A Tabletop, High-Gradient Vacuum Laser Accelerator
}

by

\author{
Frederic V. Hartemann, Anthony L. Troha, Hector A. Baldis \\ Institute for Laser Science and Application, Lawrence Livermore National Laboratory
}

\begin{abstract}
The inverse free-electron laser (IFEL) interaction is studied both theoretically and numerically in the case where the drive laser intensity approaches the relativistic regime, and the pulse duration is only a few optical cycles long. We show that by using an ultrashort, ultrahigh-intensity drive laser pulse, the IFEL interaction bandwidth and accelerating gradient are increased considerably, thus yielding large energy gains. Using a chirped pulse and negative dispersion focusing optics allows one to take further advantage of the laser optical bandwidth and produce a chromatic line focus maximizing the gradient. The combination of these novel ideas results in a compact vacuum laser accelerator capable of accelerating picosecond electron bunches with a high gradient $(\mathrm{GeV} / \mathrm{m})$ and very low energy spread. A computer code which takes into account the three-dimensional nature of the interaction is currently in development and results are expected this Spring.
\end{abstract}




\section{INTRODUCTION}

The inverse free-electron laser (IFEL) interaction has been proposed as a viable vacuum laser acceleration process $[1,2]$. Pioneering experimental work performed at Columbia University first demonstrated IFEL acceleration [3], and was followed by experiments using a nanosecond-duration, $\mathrm{GW} \mathrm{CO}_{2}$ laser at Brookhaven National Laboratory (BNL) $[4,5]$. The IFEL is attractive for laser acceleration because the interaction is mediated in a vacuum, away from boundary conditions, thus allowing for a relatively large interaction region; in addition, the absence of a plasma as an accelerating medium [6-11] resolves a number of potential problems, including plasma instabilities, nonlinear laser propagation, shot-to-shot reproducibility of the plasma, and the extremely small accelerating potential well, or "bucket," which characterizes laser-plasma acceleration schemes [12-15]. Finally, the IFEL wiggler is suitable to provide good focusing and electron beam optics and transport, thus offering the potential for producing the high-quality electron beams required for advanced light sources, biomedical applications, and the Next Linear Collider (NLC). However, one of the fundamental limitations of this acceleration scheme is the dephasing of the trapped electron with respect to the drive laser wave: As the electron energy increases, the free-electron laser (FEL) resonance condition $[16,17]$ can no longer be maintained, and the electron reaches a maximum energy given by the FEL interaction bandwidth. This problem can be addressed in two different ways: either the wiggler period and/or amplitude can be tapered $[4,18]$, as successfully demonstrated in a high-efficiency FEL [19], or, equivalently, the drive laser pulse can be chirped. The latter approach will be discussed extensively in this paper. Additionally, it should be noted that thus far, IFEL gradients 
were comparable to those possible with high-frequency rf systems (roughly up to 100 $\mathrm{MeV} / \mathrm{m})$.

By contrast, the main focus of this work is the preliminary theoretical and computational study of the IFEL interaction in a different regime, where we consider ultra-short, TW-class drive laser pulses which are now routinely generated by tabletop systems using chirped pulse amplification (CPA) [20-22]. For such femtosecond (fs) laser pulses, the IFEL interaction bandwidth is considerably wider than in the case of lower intensity drive pulses with durations in the picosecond-nanosecond range: Essentially, the well-known FEL resonance condition indicates that when the electron slips over one laser optical cycle, it also propagates over one wiggler period; thus, for the femtosecond pulses considered here, the wiggler interaction region is extremely short, and the IFEL resonance bandwidth is correspondingly wide. This directly translates into the fact that the electron energy can now vary significantly before the IFEL interaction detunes and saturates; in addition, the ultrahigh laser pulse intensity yields a high accelerating gradient. Therefore, the IFEL interaction physics is expected to change dramatically for broadband (femtosecond) drive laser pulses near the so-called relativistic intensity regime $\left(>10^{17} \mathrm{~W} / \mathrm{cm}^{2}\right.$ for optical wavelengths).

In this paper, we also show how the use of a chirped laser pulse allows the FEL resonance condition to be maintained beyond the conventional dephasing limit, thus further improving the electron energy gain. Again, we note that such laser pulses are easily produced using the CPA [20-22] technique which has led to the generation of femtosecond, multi-terawatt optical pulses, with tabletop laser systems operating at modest energies (in the joule range). We will show that the ultrashort, high-intensity 
laser pulses thus generated make it possible to design an IFEL with very high accelerating gradients $(>1 \mathrm{GeV} / \mathrm{m})$, in contrast with the longer pulse approaches previously considered. In fact, the need to alleviate electron dephasing for narrow-band laser pulses has motivated the development of period- and amplitude-tapered wigglers which help maintain resonance throughout the IFEL interaction region $\cdot[4,5,18]$, an approach which is similar and complementary to the concept presented here. Another practical limitation of IFEL accelerators is the diffraction of the drive laser pulse. In the conventional beam geometry, optical guiding [17] cannot be used because the phase shift of the IFEL interaction has the opposite sign of the FEL phase shift, which results in the well-known guiding effect. We suggest how this can be alleviated by taking advantage of the ultra-wide optical bandwidth of the chirped laser pulse: Negative dispersion focusing optics can be used to produce a chromatic line focus, where long wavelengths are focused first, while the shorter wavelengths required to maintain the FEL resonance condition at higher energies are focused further along the interaction region. The combination of these novel ideas results in a compact, efficient vacuum laser accelerator, relying on CPA and the IFEL concept, as illustrated schematically in Fig. 1. Finally, it will be shown that the accelerating IFEL bucket is very wide compared to plasma-based laser acceleration schemes [6-11]: We find that for a 1 ps FWHM (full-width at halfmaximum) Gaussian electron bunch, and a $1 \mathrm{~cm}$ period wiggler, the IFEL energy spread is $<0.9 \%$. This is extremely advantageous for a practical laser accelerator, as the device could be driven by a conventional rf photoinjector $[23,24]$. This paper is organized as follows: in Sec. II, after a brief discussion of the motivation for the chirped-pulse IFEL concept, we provide a short presentation of the analytical theory of the chirped-pulse 
IFEL interaction. In Sec. III, a simple one-dimensional computer code is described, as well as the results of simulations demonstrating the relevance of the IFEL to highgradient acceleration, and a short discussion of tapering and chirping as means to increase the IFEL interaction bandwidth. In Sec. IV, a preliminary ray-tracing study of the aforementioned chromatic line focus is outlined. Section $V$ focuses on the study of the chirped-pulse IFEL output energy spectrum, where very low energy spreads are predicted for picosecond-duration electron bunches produced by a conventional rf system. Finally, conclusions are drawn in Sec. VI.

\section{ONE-DIMENSIONAL CHIRPED-PULSE IFEL THEORY}

A brief discussion of this novel vacuum acceleration concept can be given by considering the well-known FEL resonance condition, $\lambda \approx \lambda_{w}\left(1+A_{w}^{2}\right) / 2 \gamma^{2}$, where $\lambda$ is the drive laser wavelength, $\lambda_{w}$ is the wiggler period, $A_{w}=e B_{w} \lambda_{w} / 2 \pi m_{0} c$ is the normalized vector potential of the wiggler, and $\gamma$ is the electron energy. It should be noted that in the above equation, the laser intensity is sufficiently small that its radiation pressure is negligible; in other words, the normalized vector potential of the laser satisfies the condition $A_{0}^{2}=\left(e E_{0} / \omega_{0} m_{0} c\right)^{2} \ll 1$. It is clear that, as the electron energy increases during the IFEL acceleration process, reson. nce cannot be maintained, thus saturating the interaction and limiting the energy gain. In particular, the higher the accelerating gradient, the faster dephasing occurs; however, for ultrashort laser pulses, this is balanced by the increased interaction bandwidth. To alleviate this problem, it has been proposed to taper the wiggler amplitude, $B_{w}$, or the wiggler period, $\lambda_{w}[4,5,18]$. This approach has 
proved extremely successful in the case of FELs, as exemplified by the results obtained by Orzechowski et al., at Lawrence Livermore National Laboratory (LLN̦L).[19]; however, one of the key requirements for the implementation of a tapered wiggler is the adiabaticity of the tapering, in order to preserve the electron beam quality. Clearly, for the very intense drive laser pulses necessary for high IFEL gradients, this requirement is difficult to meet, as the entire IFEL interaction now becomes strongly nonlinear and nonadiabatic. In contrast, with our technique, the drive laser wavelength, 1 , decreases as the electron gains energy, thus maintaining resonance beyond the aforementioned saturation length. Such chirped, ultrahigh-intensity laser pulses are easily produced using CPA, and tabletop systems with high repetition rates $(20-1000 \mathrm{~Hz})$ are now available [25-27]. We now present a short theoretical analysis of the chirped-pulse IFEL interaction. In this analysis, length is measured in units of the central laser wavelength, $1 / k_{0}=\lambda_{0} / 2 \pi$, time in units of the laser frequency, $1 / \omega_{0}$, mass in units of $m_{0}$, and charge in units of $e$. For the sake of simplicity, plane waves are considered. Here, the combined four-potential of the drive laser pulse, $\mathbf{A}_{\ell}(\phi)$, and the wiggler magnetic field, $\mathbf{A}_{w}(z)$, is given by

$$
A_{\mu}=\left(\varphi, \mathbf{A}_{\perp}, A_{z}\right), \quad \varphi=A_{z}=0, \quad \mathbf{A}_{\perp}\left(x_{\mu}\right)=\mathbf{A}_{c}(\phi)+\mathbf{A}_{w}(z),
$$

where we have introduced the phase of the traveling laser wave, $\phi=k_{\mu} x^{\mu}=t-z$. The corresponding electromagnetic field components are

$$
\mathbf{E}_{\perp}=-\frac{\partial \mathbf{A}_{\perp}}{\partial t}=-\frac{d \mathbf{A}_{\ell}}{d \phi}, \quad \mathbf{B}_{\perp}=\nabla \times \mathbf{A}_{\perp}=\hat{z} \times\left(\mathbf{E}_{\perp}+\frac{d \mathbf{A}_{w}}{d z}\right)
$$


Neglecting radiation reaction [28-30], the Lorentz force equation governs the evolution of the electron four velocity, $u_{\mu}=d x_{\mu} / d \tau$, where $\tau$ is the electron proper time, which, in our units, corresponds to the energy-momentum four vector. We thus have, for the transverse motion,

$$
\frac{d \mathbf{u}_{\perp}}{d \tau}=-\left(\gamma \mathbf{E}_{\perp}+\hat{z} u_{z} \times \mathbf{B}_{\perp}\right)=\left(\gamma-u_{z}\right) \frac{d \mathbf{A}_{\ell}}{d \phi}+u_{z} \frac{d \mathbf{A}_{w}}{d z}
$$

where we recognize the light-cone variable, $\kappa=d \phi / d \tau=\gamma-u_{z}$. Equation (3) can be readily integrated to yield the transverse momentum invariant: $\mathbf{u}_{\perp}=\mathbf{A}_{\iota}(\phi)+\mathbf{A}_{w}(z)[16$, 17]. The evolution of the electron energy and axial momentum are given by

$$
\frac{d \gamma}{d \tau}=-\mathbf{u}_{\perp} \cdot \mathbf{E}_{\perp}=\frac{1}{2} \frac{d \mathbf{A}_{\ell}^{2}}{d \phi}+\mathbf{A}_{w} \cdot \frac{d \mathbf{A}_{\ell}}{d \phi},
$$

and

$$
\frac{d u_{z}}{d \tau}=-\mathbf{u}_{\perp} \times \mathbf{B}_{\perp}=\frac{1}{2} \frac{d \mathbf{A}_{t}^{2}}{d \phi}+\frac{1}{2} \frac{d \mathbf{A}_{w}^{2}}{d z}-\mathbf{A}_{t} \cdot \frac{d \mathbf{A}_{w}}{d z}-\mathbf{A}_{w} \cdot \frac{d \mathbf{A}_{t}}{d \phi},
$$

where we recognize the laser radiation pressure (ponderomotive potential) $d \mathbf{A}_{c}^{2} / d \phi$, and the IFEL interaction terms, involving the nonlinear product of the laser and wiggler vector potentials. In the case of a helically polarized wiggler with constant amplitude, $d \mathbf{A}_{w}^{2} / d z$ is identically zero. The IFEL acceleration gradient scaling is given by

$$
\frac{d \gamma}{d z}=\frac{d \gamma}{d \tau} \frac{d \tau}{d z}=\frac{\mathbf{A}_{w}}{u_{z}} \cdot \frac{d \mathbf{A}_{l}}{d \phi}
$$


thus indicating that high acceleration gradients can be obtained by using ultrahighintensity drive laser pulses, where both the normalized laser and wiggler vector potentials approach unity. The equation governing the evolution of the light-cone variable is obtained by subtracting the axial force equation from the energy equation, to yield

$$
\frac{d \kappa}{d \tau}=\frac{d}{d \tau}\left(\gamma-u_{z}\right)=-\mathbf{u}_{\perp} \cdot \mathbf{E}_{\perp}+\mathbf{u}_{\perp} \times \mathbf{B}_{\perp}=\mathbf{u}_{\perp} \cdot \frac{d \mathbf{A}_{w}}{d z}
$$

Using the result obtained for the transverse momentum, and for a helical wiggler, Eq. (7) reduces to

$$
\frac{d \kappa}{d \tau}=\mathbf{A}_{\ell} \cdot \frac{d \mathbf{A}_{w}}{d z}
$$

This equation is particularly simple, and it indicates that if either the wiggler or the laser field is zero, the light-cone variable is invariant. The axial momentum and energy can now be expressed in terms of the light-cone variable as

$$
\gamma=\frac{1+\mathbf{A}_{\perp}^{2}+\kappa^{2}}{2 \kappa}, \quad u_{z}=\frac{1+\mathbf{A}_{\perp}^{2}-\kappa^{2}}{2 \kappa} .
$$

Since $\lim _{\phi \rightarrow \pm \infty}\left|\mathbf{A}_{c}(\phi)\right|$ and $\lim _{z \rightarrow \pm \infty}\left|\mathbf{A}_{w}(z)\right|$, it is clear that the electron will have a net energy gain only if the light-cone variable is larger at the end of the interaction. We also note that, during the interaction, the energy can be increased locally by the laser radiation pressure or the IFEL interaction [29]. This so-called "ponderomotive" acceleration process $[29,31,32]$ has been proposed as a laser acceleration scheme; however, a viable concept for the extraction of the accelerated electrons from the laser pulse proves extremely difficult to envişion: The termination of the interaction necessarily involves a 
boundary condition which modifies the laser pulse characteristics and it completely degrades the accelerated electron beam quality. It proves useful to consider the electron phase in the laser pulse as the independent variable; in this case, we have

$$
\frac{d \kappa}{d \phi}=\frac{d \kappa}{d \tau} \frac{d \tau}{d \phi}=\frac{d}{d \tau}[\ln (\kappa)]=\frac{\mathbf{A}_{\ell}}{\kappa} \cdot \frac{d \mathbf{A}_{w}}{d z}
$$

and the electron position is given by

$$
x_{\mu}(\phi)=\int_{0}^{\phi} \frac{u_{\mu}(\psi)}{\kappa(\psi)} d \psi
$$

Because we are considering ultrashort chirped drive laser pulses, nonlinear slippage must be taken into account, as well as the diffraction (Rayleigh) length of the focused laser pulse. The nonlinear slippage length is given by the $z$ component of Eq. (11). For such pulses, the slippage length can be much longer than the laser pulse itself, thus allowing long electron bunches to interact in the IFEL. This effect is discussed and demonstrated numerically in Sec. V.

\section{CHIRPED-PULSE IFEL COMPUTER SIMULATIONS}

Based on the theoretical model described above, we have developed a simple, one-dimensional, honlinear computer code which follows the evolution of the light-cone variable as a function of the electron phase within the drive laser pulse. This onedimensional analysis gives an adequate first-order description of the IFEL interaction physics provided that the drive laser pulse can be modeled by a plane wave, which implies a large $f$ number and a long Rayleigh range, and as long as the IFEL electron 
trajectories are confined to a region where the transverse gradient of the wiggler and laser fields are small. Three-dimensional effects will be studied using a more sophisticated interaction code, currently under development. Specifically, the vector potential of the laser is modeled by a circularly polarized plane wave with a linear chirp, $\alpha$,

$$
\mathbf{A}_{\ell}(\phi)=A_{0} g(\phi)\left[\hat{x} \sin \left(\phi+\alpha \phi^{2}\right)+\hat{y} \cos \left(\phi+\alpha \phi^{2}\right)\right]
$$

The pulse envelope is chosen as $g(\phi)=\sin ^{2}[\phi / \Delta \phi]$, which closely approximates a Gaussian near the maximum, and has a finite duration. Here, $\Delta \phi$ is the pulse FWHM, measured in units of $1 / \omega_{0}$, and $A_{0}$ is the amplitude of the laser pulse measured in units of $m_{0} c / e$. The helical wiggler field is simply given by

$$
\mathbf{A}_{w}(z)=A_{w}\left[\hat{x} \sin \left(k_{w} z\right)+\hat{y} \cos \left(k_{w} z\right)\right]
$$

where the wiggler wave number is given in the units chosen here by $k_{w}=\lambda_{0} / \lambda_{w}$. The position of the electron in the wiggler is given by Eq. (11) as $z(\phi)=\int_{0}^{\phi} u_{z}(\psi) / \kappa(\psi) d \psi$. Finally, in order to increase the accuracy of the code, a second-order Runge-Kutta algorithm is used; for this purpose, we evaluate the second-order derivative of the lightcone variable with respect to phase,

$$
\frac{d^{2} \kappa}{d \phi^{2}}=\frac{1}{\kappa}\left[\frac{d \mathbf{A}_{t}}{d \phi} \cdot \frac{d \mathbf{A}_{w}}{d z}-k_{w}^{2} \frac{d z}{d \phi}\left(\mathbf{A}_{\epsilon} \cdot \mathbf{A}_{w}\right)-\left(\frac{d \kappa}{d \phi}\right)^{2}\right] .
$$


The accuracy of the code is verified by comparing two different values of the energy, namely,

$$
\gamma(\phi)=\gamma(\phi=0)+\int_{0}^{\phi} \frac{1}{\kappa(\phi)}\left[\frac{1}{2} \frac{d \mathbf{A}_{\ell}^{2}(\psi)}{d \phi}+\mathbf{A}_{w}(\psi) \cdot \frac{d \mathbf{A}_{\ell}(\psi)}{d \phi}\right] d \psi
$$

and

$$
\gamma(\phi)=\sqrt{1+\mathbf{u}_{\perp}^{2}(\phi)+u_{z}^{2}(\phi)}
$$

The relative numerical error is obtained by dividing the difference between Eqs. (15) and (16) by the average value of the energy. To demonstrate the relevance of the chirpedpulse IFEL concept, we ran the code for the following parameters: The normalized vector potential of the drive laser pulse is $A_{0}=0.2$, the pulse duration is $10 \mathrm{fs}$, the central laser wavelength is $\lambda_{0}=0.8 \mu \mathrm{m}$, the wiggler field is $B_{w}=2 \mathrm{kG}$, the wiggler period is $\lambda_{w}=1 \mathrm{~cm}$, and the injection energy is $\gamma_{0}=65$, while the resonant IFEL interaction energy is $\gamma^{*}=80$. The results are shown in Fig. 2 for both an unchirped laser pulse, and an optimally chirped laser pulse, where $\alpha=0.007$. In the first case, the electron clearly detunes away from the IFEL resonance, and exits the interaction region with an energy gain corresponding to an average acceleration gradient of $0.343 \mathrm{GeV} / \mathrm{m}$. This number is still quite high because we are using an. ultrashort laser pulse with a high focused intensity: the intensity can be expressed as

$$
I_{0}=\left(\frac{A_{0}}{\lambda_{0}}\right)^{2}\left(\frac{2 \pi^{2} \varepsilon_{0} m_{0}^{2} c^{5}}{e^{2}}\right)=\left(\frac{A_{0}}{\lambda_{0}}\right)^{2} 13.68 \mathrm{GW},
$$


which corresponds to $I_{0}=8.55 \times 10^{16} \mathrm{~W} / \mathrm{cm}^{2}$ for the parameters given above. At this point, it is important to understand that attempts to decouple the electron at its peak energy will result in a severe loss of beam quality. In the second case, however, the IFEL resonance is maintained throughout the interaction region, and the electron energy now corresponds to an average gradient of $0.567 \mathrm{GeV} / \mathrm{m}$, or a $65 \%$ increase over the unchirped case. More importantly, in this case, no extraction scheme is required, thus preserving the accelerated beam quality. The normalized wavelength of the chirped pulse can be given as a function of the electron position in the interaction region by plotting $1 /(1+\alpha \phi)$ versus $z(\phi)$, as shown in Fig. 3. The relative optical bandwidth of the chirp is found to be $15 \%$, which corresponds to $120 \mathrm{~nm}$ of spectral width. The bandwidth of the $10 \mathrm{fs}$ pulse, including the additional $15 \%$ chirp, is, therefore, approximately $200 \mathrm{~nm}$, or 5 fs transform-limited duration. This value has been demonstrated experimentally for $\mathrm{Ti}: \mathrm{Al}_{2} \mathrm{O}_{3}$ systems $[33,34]$. The code has also been used to model a tapered wiggler IFEL; we find that using the same parameters, but without a chirped laser pulse, results in a similar gradient. This gradient is possible with an increase of the wiggler period from 1 to $1.3 \mathrm{~cm}$ over four periods because of the inherently large bandwidth of the $10 \mathrm{fs}$ drive laser pulse; the amplitude of the wiggler remains constant over the interaction region. Therefore, tapering and chirping should be considered as complementary means to improve the IFEL gradient; both methods will be combined in a proof-of-principle experimental design. The parameters discussed above can be translated into experimental parameters by assuming a long cylindrical focus for the drive laser pulse: Using a Rayleigh range $z_{0}=\pi\left(w_{0}^{2} / \lambda_{0}\right)=2 \mathrm{~cm}$, the focal waist is $w_{0}=71.4 \mu \mathrm{m}$. The corresponding peak laser power is $P_{0}=l_{0} \pi w_{0}^{2}=l_{0} \lambda_{0} z_{0}=13.7 \mathrm{TW}$, and the laser pulse 
energy takes the modest value of $W_{0}=P_{0} \Delta t=137 \mathrm{~mJ}$; this is considerably lower than the tens of joules commonly used in conventional rf systems to power accelerating sections of a few tens of mega-electron-volts. In fact, it is interesting to compare the energy in the drive laser pulse to the kinetic energy acquired by a $1 \mathrm{nC}$ electron bunch in the IFEL: We find that the energy transferred to the beam is $22.7 \mathrm{~mJ}$, or $16.6 \%$ of the laser energy. This shows that the IFEL can be significantly loaded, which is key to efficient acceleration. It is also interesting to evaluate the radius of the electron trajectory in the helical wiggler: we have $k_{w} r_{\perp}=u_{\perp} / u_{\|} \leq A_{w} / \gamma_{0}$, and the wiggler parameter $A_{w}=0.18$; therefore, $r_{\perp}=4.4 \mu \mathrm{m} \ll w_{0}$, and the one-dimensional model should appropriately describe the IFEL interaction. Clearly, this represents a worst-case scenario, as a linearly polarized laser and wiggler and a slab focus could be used; in addition, it is important to note that the intensity of the laser along the focal region can be maximized by using an optical scheme that will provide a chromatic line focus. The long wavelength part of the pulse will be focused closer to the optics, with the shorter wavelengths focused further down the propagation axis. This can be accomplished in a number of ways, using either reflective, refractive, or diffractive optics. An interesting and simple approach is to use a Fresnel zone plate, which will produce the required $d \lambda / d z$ to match the computer calculation presented earlier. This approach is expected to relax the drive laser energy requirement by one order of magnitude. 


\section{CHROMATIC LINE FOCUS}

We have performed a preliminary ray-tracing study of the chromatic line focus using OPTICA [35]; the results are illustrated in Fig. 4, where the optical setup is shown schematically (top), as well as the resulting focal spot position as a function of the wavelength (bottom). The focusing system consists of a lens pair, including a biconvex lens made of flint glass, and a plano-concave lens made of crown glass, which is the opposite of a conventional achromatic doublet [36]. The materials chosen here are zinc selenide for the flint and BK7 for the crown. The group velocity dispersion (GVD) introduced by the doublet can be recompensated for in the CPA stretcher, following the approach pioneered by Barty et al. [25]. The chromatic line focus thus obtained is shown in Fig. 4 (bottom) and compared to the chirped-pulse IFEL simulation; the results are well matched to first order, and demonstrate the validity of this approach. The design of higher-order line focusing optics will be performed in the near future. The Rayleigh range of this system can be compared to the value chosen in our earlier discussion of experimental parameters, presented in Sec. III, to indicate the advantage of the chromatic line focus over a conventional focusing system: The beam diameter used in our OPTICA runs is $d=4 \mathrm{~cm}$, and the focal length of the doublet is $f=1 \mathrm{~m}$; this yields a convergence angle $\theta=d / 2 f=\lambda_{0} / \pi w_{0}=20 \mathrm{mrad}$, which translates into a focal spot size $w_{0}=25.4 \mu \mathrm{m}$ and a Rayleigh range equal to $2.54 \mathrm{~mm}$, which is much smaller than the value of $2 \mathrm{~cm}$ required to span the IFEL interaction region. The ratio of the laser energy required in the case of this chromatic line focus to the previous value is approximately given by the ratio of the Rayleigh ranges, which corresponds to the ratio of the focus cross-section: $13 \%$ only, or $17.4 \mathrm{~mJ}$; the energy gain is close to one order of magnitude 
in this preliminary study. For optical wavelengths, this line focus technique appears to be easier to implement than an over-moded waveguide, which has been developed and demonstrated at $\mathrm{BNL}$ for a $10.6 \mathrm{~mm} \mathrm{CO}$ laser $[4,5]$; in particular, the chromatic focus will scale more favorably to the required high intensities for high-gradient IFEL operation. A number of important issues will be addressed in a more detailed design study of a chirped-pulse IFEL chromatic line focus, including GVD and B-integral effects in the chromatic focusing doublet. The three-dimensional modeling of the chromatic line focus for an ultrawideband, high-intensity laser pulse will be achieved by modifying our paraxial propagator method [37], which includes axial electromagnetic field components and satisfies the gauge condition exactly. These three-dimensional field distributions will then be used in conjunction with a relativistic code to ascertain the stability and quality of the chirped-pulse IFEL acceleration mechanism.

\section{CHIRPED-PULSE IFEL ENERGY SPECTRUM}

We have performed a detailed study of the acceptance of the IFEL accelerating bucket. This is shown in Fig. 5, where the IFEL energy gain is given as a function of the initial position in the wiggler, which is defined as the point where the laser pulse

overtakes the electron $[z(\phi=0)]$. The periodicity, which is clearly seen, results from the one-dimensional nature of our model: In the absence of diffraction, the IFEL is invariant by translation of $\lambda_{w}$. It is also important to note here that although both the drive laser pulse and the electron bunch are quite short, the nonlinear IFEL slippage length is a few centimeters, thus explaining why a picosecond electron bunch can be accelerated by a femtosecond laser pulse: In the present one-dimensional model, the 
phase of the ponderomotive force is determined by the initial electron position in the wiggler, as defined above; because the IFEL is a fast-wave device, the acceptance of the accelerating bucket scales with the wiggler period. This should not be confused with the bunching which occurs at the IFEL resonant wavelength. The width of the energy peak, in the chirped case, is seen to be approximately $2 \mathrm{~mm}$ FWHM. The fact that the IFEL bucket scale is given by the wiggler period directly results from the fast-wave nature of the IFEL interaction. This number is extremely large compared to the accelerating bucket in a laser-plasma system [10-15], which is roughly equal to a tenth of the plasma wavelength (typically, in the micron range); this number is also compatible with conventional rf injectors, as is demonstrated in Fig. 6 (bottom). Here, we inject a 1 ps FWHM Gaussian electron bunch into the IFEL, at the optimum phase $(0.2 \mathrm{~mm})$ for maximum energy gain, as shown in Fig. 6 (top); the output electron energy spectrum is then computed, and we find nearly $60 \%$ of the accelerated charge in a $0.125 \mathrm{MeV}$ bin at $57.8 \mathrm{MeV}$. This represents an energy spread of $<0.2 \%$; furthermore, $90 \%$ of the accelerated charge is found within a $0.5 \mathrm{MeV}$ energy interval, which corresponds to a spread smaller than $0.9 \%$. The $32.8 \mathrm{MeV}, 1$ ps electron bunch injected in the IFEL could be produced by an X-band plane wave transformer linac (PWT) very similar to that currently developed by DULY Research, Incorporated [23, 24]. We also note that the IFEL resonance cundition indicates that high-energy (tera-electron-volt) scaling is possible, at least in principle: Assuming that the wiggler magnetic field scales linearly with the wiggler wavelength, and using a value of $\chi=2 \mathrm{kG} / \mathrm{cm}$ for the scaling, which is easily achievable with current technology, the resonance condition can be recast as $\gamma \approx\left(\lambda_{w} / \lambda^{*}\right)^{5 / 2}$, where the length scale $\lambda^{*}=\left[2 \pi\left(\lambda_{0} / r_{0}\right) m_{0} / \varepsilon_{0} \chi^{2}\right]^{1 / 5}=3.4066 \mathrm{~mm}$; here 
$r_{0}=2.8178 \times 10^{-15} \mathrm{~m}$, is the classical electron radius, and we have used $\lambda_{0}=800 \mathrm{~nm}$. For $\lambda_{w}=1 \mathrm{~m}$, the resonant IFEL energy is $0.754 \mathrm{TeV}$. Naturally, parasitic effects, such as synchrotron radiation losses, must be taken into account to further assess the highenergy scalability of the chirped-pulse IFEL. Finally, in the limit of high energies (gigaelectron-volts-tera-electron-volts) and ultrahigh intensities, well into the relativistic regime $\left(A_{0}>1\right)$, the IFEL concept has been studied theoretically by a few authors [3842]; their results confirm that a static magnetic field can help dephase the accelerated electrons with respect to the drive laser pulse, thus yielding net energy gain even in the case of a plane wave interaction. Furthermore, these studies also show that the reversal of the static field allows one to maintain the accelerating gradient, as in an IFEL, and that for high gradients, the magnetic field should be tapered every half period. This approach, which closely parallels a tapered wiggler, proves difficult to implement in a practical device, as discussed earlier, but the combination of chirped drive laser pulses, and wiggler sections of increasing period and field strength might be the right scheme to stage an IFEL accelerator to high energies.

\section{CONCLUSIONS}

In conclusion, we have presented a new concept for high gradient laser acceleration in vacuum: the chirped-pulse IFEL. The essential idea is to use an ultrashort (femtosecond), ultrahigh-intensity laser pulse to increase both the IFEL interaction bandwidth and accelerating gradient, thus yielding large energy gains in a compact system. In addition, we have shown that the IFEL resonance condition can be 
maintained throughout the interaction region by using a chirped drive laser wave, where the accelerated electron first interacts with the red part of the spectrum, and then progressively with the blue end of the spectrum as it slips nonlinearly within the phase of the drive pulse. We have demonstrated the relevance of this concept by using a onedimensional nonlinear code indicating that accelerating gradients near $1 \mathrm{GeV} / \mathrm{m}$ are predicted with modest laser and beam parameters, and that the use of a chirped pulse results in a $65 \%$ improvement of the average gradient. In addition, we have performed a detailed study of the acceptance of the IFEL accelerating bucket and found that it is wider than the plasma-wakefield accelerating potential well by two to three orders of magnitude; as a result, picosecond electron bunches can be accelerated with extremely low energy spread, as opposed to the femtosecond bunches required by most laser-plasma acceleration schemes [10-15]. Finally, we have suggested how diffraction could be alleviated by taking advantage of the laser optical bandwidth with negative dispersion focusing optics to produce a chromatic line focus. The combination of these novel ideas results in a compact, efficient vacuum laser accelerator. The new techniques that we have brought to bear on the problem of creating a viable IFEL, a preliminary account of which was published in Reference [43], have been well-received in the literature [44]. A patent application for the chirped-pulse IFEL scheme has been filed through Lawrence Livermore National Laboratory. Current work includes the development of a fully threedimensional interaction code, and extensive ray tracing and design of the negative dispersion focusing optics required to produce a line focus with the appropriate geometry for chirped-pulse IFEL acceleration, as well as a detailed design study of the wiggler interaction region and electron beam optics. 


\section{References}

1 R. B. Paimer, J. Appl. Phys. 43, 3014 (1972).

2 E. D. Courant, C. Pellegrini, and W. Zakowicz, Phys. Rev. A 32, 2813 (1985).

3 I. Wernick and T. C. Marshall, Phys. Rev. A 46, 3566 (1992).

4 A. Van Steenbergen, J. Gallard, J. Sandweiss, and J.-M. Fang, Phys. Rev. Lett. 77, 2690 (1996).

5 A. Van Steenbergen and J. C. Gallardo, Proceedings of the 1997 Particle Accelerator Conference (1998), p.616.

6 C. Joshi, T. Tajima, J. M. Dawson, H. A. Baldis, and N. A. Ebrahim, Phys. Rev. Lett. 47, 1285 (1981).

7 C. E. Clayton, K. A. Marsh, A. Dyson et al., Phys. Rev. Lett. 70, 37 (1993).

8 D. P. Umstadter et al., Science 273, 474 (1996).

9 H. M. Milchberg et al., Phys. Plasmas 3, 2149 (1996).

10 P. Sprangle, E. Esarey, and J. Krall, Phys. Plasmas 3, 2183 (1996).

11 E. Esarey et al., IEE.¿ Trans. Plasma Sci. 24, 252(1996).

12 D. Umstadter, J. K. Kim, and E. Dodd, Phys. Rev. Lett. 76, 2073 (1996).

13 E. Esarey, R. F. Hubbard, W. P. Leemans, A. Ting, and P. Sprangle, Phys. Rev. Lett. 79, 2682 (1997).

14 W. P. Leemans, P. Volfbeyn, K. Z. Guo et al., Phys. Plasmas 5, 1615 (1998). 
15 E. Esarey et al., in Ref. 5, p. 630.

16 T. C. Marshall, Free-Electron Lasers (McMillan, New York, 1985).

17 C. W. Roberson and P. Sprangle, Phys. Fluids B 1, 3 (1989).

18 G. T. Moore, Phys. Rev. Lett. 60, 1825 (1988).

19 T. J. Orzechowshi, B. R. Anderson, J. C. Clark et al., Phys. Rev. Lett. 57, 2172 (1986).

20 M. D. Perry and G. Mourou, Science 264, 917 (1994).

21 G. Mourou, C. P. J. Barty, and M. D. Perry, Phys. Today 51 (1), 22 (1998).

22 D. P. Umstadter et al., Opt. Photonics News 9, 41 (1998).

23 D. Yu et al., in Ref. 5, p. 2802.

24 D. Yu et al., Abs. 1999 Particle Accelerator Conference (1999), p.118.

25 C. P. J. Barty, C. L. Gordon III, and B. E. Lemoff, Opt. Lett. 19, 1442 (1994).

26 J. Zhou et al., Opt. Lett. 20, 64 (1995).

27 A. Sullivan et al., Opt. Lett. 21, 603 (1996).

28 P. A. M. Dirac, Proc. R. Soc. London, Ser. A 167, 148 (1938).

29 F. V. Hartemann, Phys. Plasmas 5, 2037 (1998).

30 F. V. Hartemann and A. K. Kerman, Phys. Rev. Lett. 76, 624 (1996).

31 F. V. Hartemann, J. R. Van Meter, A. L. Troha et al., Phys. Rev. E 58, 5001 (1998).

32 B. Quesnel and P. Mora, Phys. Rev. E 58, 3719 (1998).

33 M. Nisoli et al., Opt. Lett. 22, 522 (1997).

34 U. Morgner et al., Opt. Lett. 24, 411 (1999).

35 D. Barnhart, OPTICA, Optica Software, Urbana, IL, 1995.

36 M. Born and E. Wolf, Principles of Optics (Pergamon, Oxford, 1980).

37 See Ref. 29 and 31. 
38 S. Kawata, T. Maruyama, H. Watanabe, and I. Takahashi, Phys. Rev. Lett. 66, 2072 (1991).

39 M. S. Hussein and M. P. Pato, Phys. Rev. Lett. 68, 1136 (1992).

40 M. S. Hussein, M. P. Pato, and A. K. Kerman, Phys. Rev. A 46, 3562 (1992).

41 J. X. Wang et al., Phys. Lett. A 231, 139 (1997).

42 J. X. Wang, Y. K. Ho, Q. Kong, L. J. Zhu, L. Feng, S. Scheid, and H. Hora, Phys. Rev. E 58, 6575 (1998).

43 F. V. Hartemann et al., Phys. Plasma 6, 4104 (1999).

44 N. Marquardt, Nuc. Instrum. Methods A 455, 70 (2000).

\section{Figure Captions}

FIG. 1. Top: schematic of the chirped-pulse IFEL acceleration concept. Bottom: schematic of the negative dispersion, chromatic line focus.

FIG. 2. Electron normalized energy as a function of its phase within the drive laser pulse. $A_{0}=0.2,10 \mathrm{fs}$ FWHM, $\lambda_{0}=0.8 \mu \mathrm{m}, B_{w}=2 \mathrm{kG}, \lambda_{w}=1 \mathrm{~cm}, \gamma_{0}=65$.

FIG. 3. Normalized chirped-pulse wavelength as a function of position in the interaction region.

FIG. 4. Optica ray-tracing results for a chromatic line focus; details are given in the text.

FIG. 5. IFEL energy gain versus initial electron position in the wiggler.

FIG. 6. Top: initiai electron bunch charge density and IFEL energy gain as a function of the initial posii.on. Bottom: IFEL output energy spectrum for a 1 ps FWHM Gaussian electron bunch injected at the optimum phase angle; the other parameters are the same as in Fig. 2. 

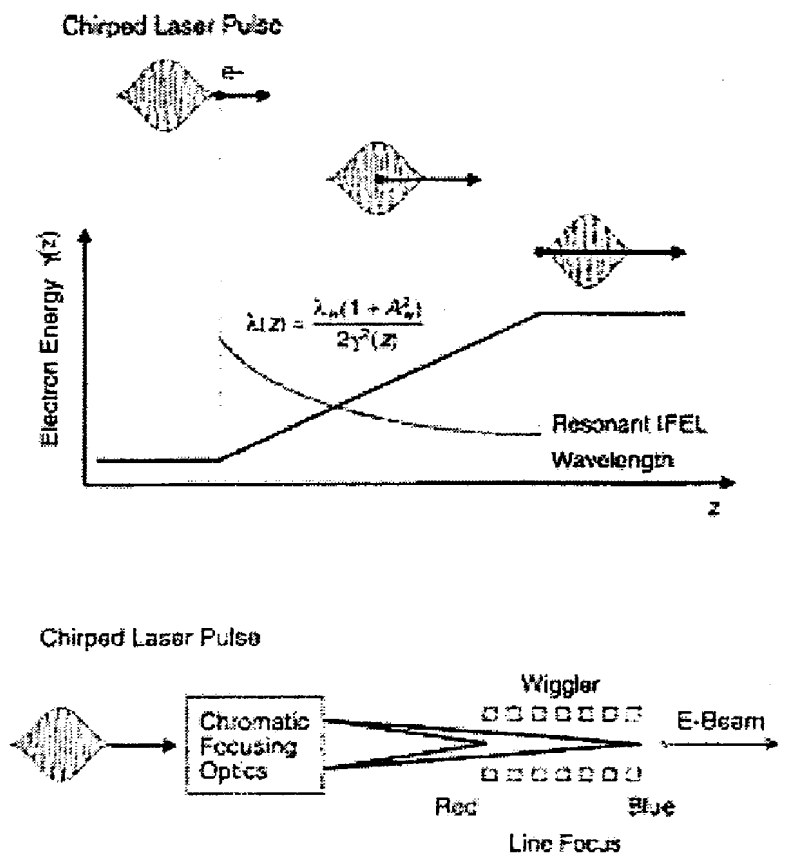

Figure 1

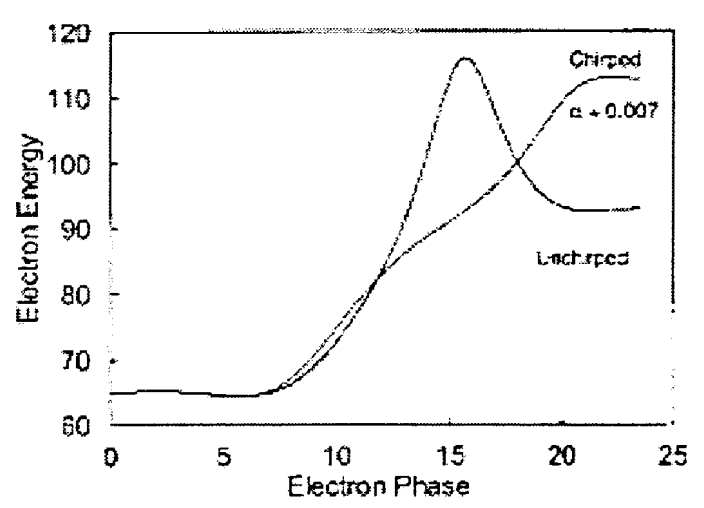

Figure 2 


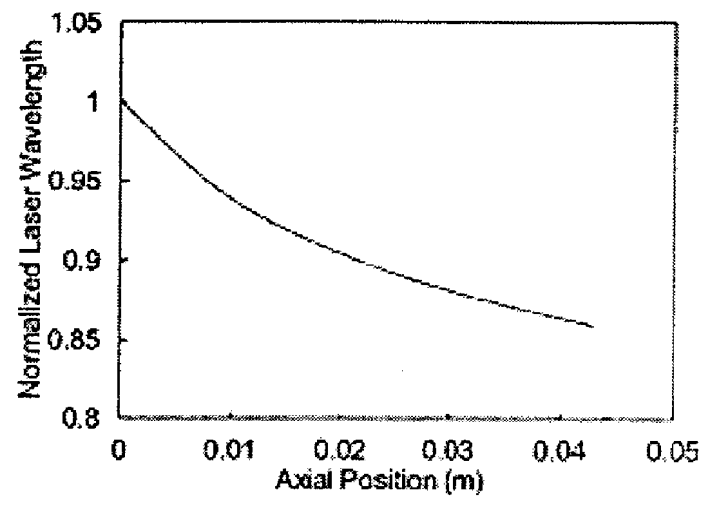

Figure 3
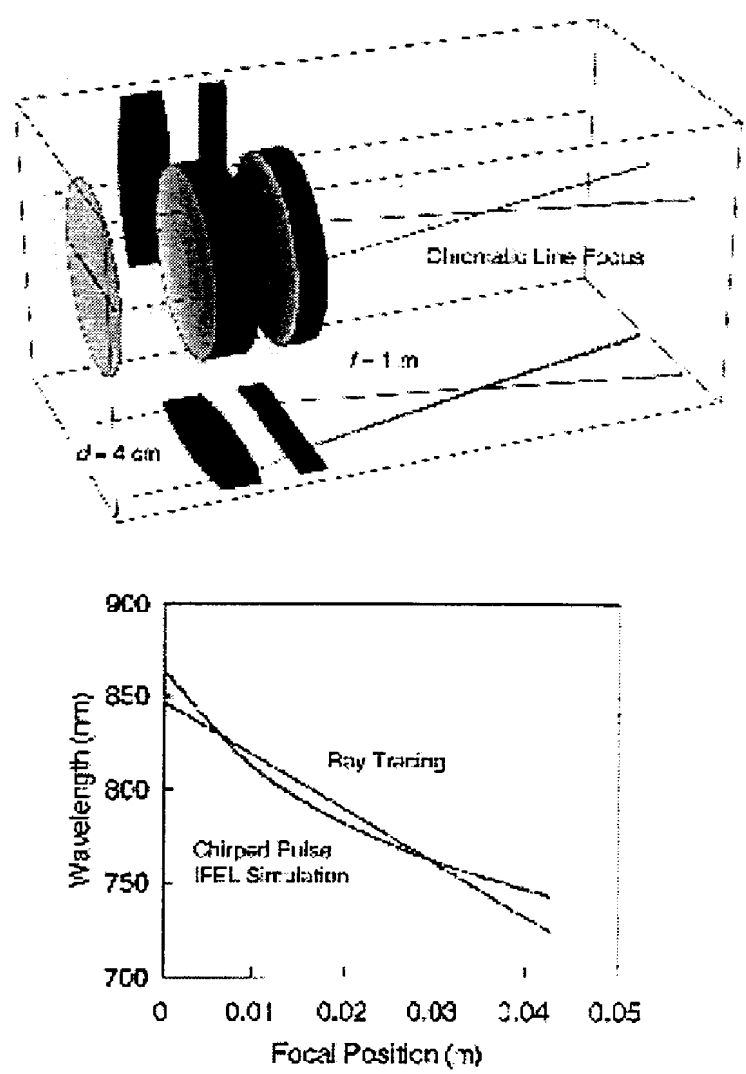

Figure 4 


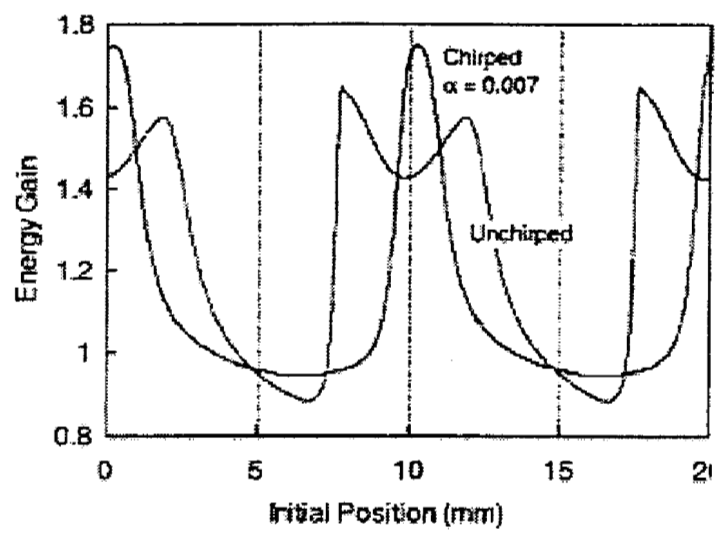

Figure 5
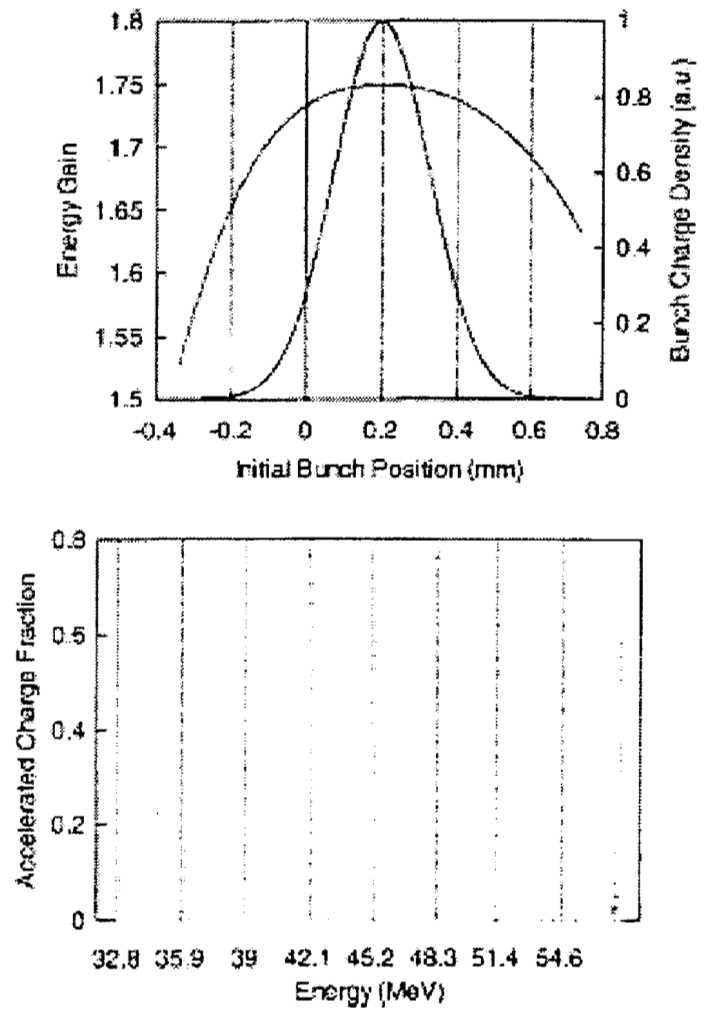

Figure 6 
University of California

Lawrence Livermore National Laboratory

Technical Information Department

Livermore, CA 94551

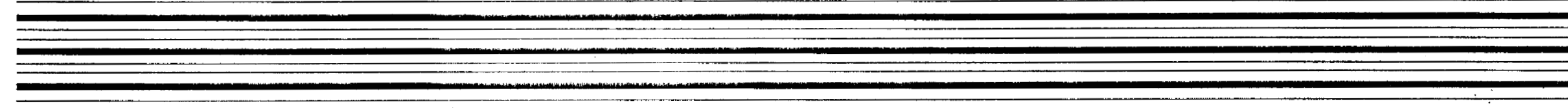

